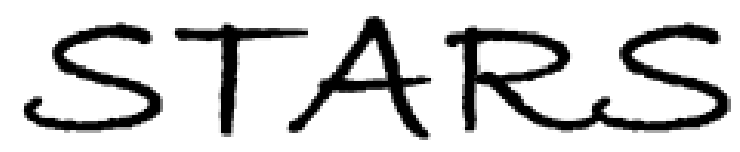

University of Central Florida

STARS

$1-1-2011$

\title{
Hanbury Brown and Twiss correlations of Anderson localized waves
}

\author{
Y. Lahini \\ Y. Bromberg \\ Y. Shechtman \\ Alexander Szameit \\ Demetrios N. Christodoulides \\ University of Central Florida
}

See next page for additional authors

Find similar works at: https://stars.library.ucf.edu/facultybib2010

University of Central Florida Libraries http://library.ucf.edu

This Article is brought to you for free and open access by the Faculty Bibliography at STARS. It has been accepted for inclusion in Faculty Bibliography 2010 s by an authorized administrator of STARS. For more information, please contactSTARS@ucf.edu.

\section{Recommended Citation}

Lahini, Y.; Bromberg, Y.; Shechtman, Y.; Szameit, Alexander; Christodoulides, Demetrios N.; Morandotti, R.; and Silberberg, Y., "Hanbury Brown and Twiss correlations of Anderson localized waves" (2011). Faculty Bibliography 2010s. 1515.

https://stars.library.ucf.edu/facultybib2010/1515

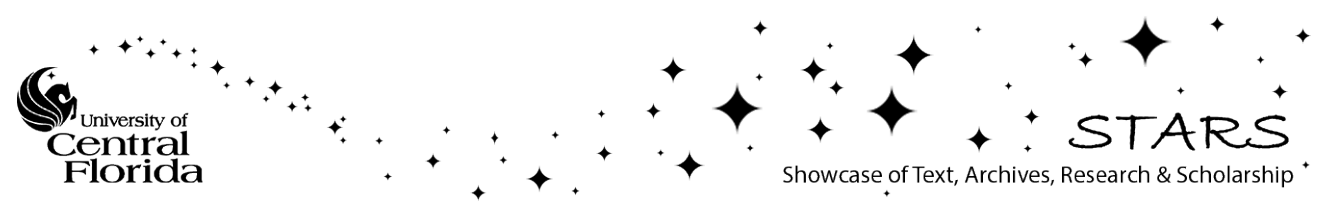


Authors

Y. Lahini, Y. Bromberg, Y. Shechtman, Alexander Szameit, Demetrios N. Christodoulides, R. Morandotti, and Y. Silberberg 


\title{
Hanbury Brown and Twiss correlations of Anderson localized waves
}

\author{
Y. Lahini, ${ }^{1}$ Y. Bromberg, ${ }^{1}$ Y. Shechtman, ${ }^{2}$ A. Szameit,${ }^{2}$ D. N. Christodoulides,${ }^{3}$ R. Morandotti, ${ }^{4}$ and Y. Silberberg ${ }^{1}$ \\ ${ }^{1}$ Department of Physics of Complex Systems, Weizmann Institute of Science, Rehovot, Israel \\ ${ }^{2}$ Department of Solid State Physics, Technion, Israel \\ ${ }^{3}$ CREOL/College of Optics, University of Central Florida, Orlando, Florida, USA \\ ${ }^{4}$ Institute National de la Recherche Scientifique, Varennes, Qubec, Canada
}

(Received 4 April 2011; published 24 October 2011)

\begin{abstract}
When light waves propagate through disordered photonic lattices, they can eventually become localized due to multiple scattering effects. Here we show experimentally that while the evolution and localization of the photon density distribution is similar in the two cases of diagonal and off-diagonal disorder, the density-density correlation carries a distinct signature of the type of disorder. We show that these differences reflect a symmetry in the spectrum and eigenmodes that exists in off-diagonally disordered lattices but is absent in lattices with diagonal disorder.
\end{abstract}

DOI: 10.1103/PhysRevA.84.041806

PACS number(s): 42.25.Dd, 42.50.Ar, 72.15.Rn

The propagation of quantum-mechanical waves in periodic and disordered media is a fundamental theme in solid state physics, underlying the transport properties of condense matter systems. In a perfectly periodic system, the translational invariance gives rise to extended eigenmodes known as the Bloch modes. As a result, in periodic systems an initially narrow wave packet will expand indefinitely and ballistically (i.e., its width will grow linearly in time). Disorder in an otherwise perfectly periodic lattice breaks the translational symmetry and can lead to exponential localization of the system's eigenmodes and to the arrest wave-packet expansion (or diffusion), a phenomenon known as Anderson localization $[1,2]$.

Traditionally, the localization of waves inside the medium was not observed directly, but rather inferred indirectly from transmission or conductance measurements. Recently, a new approach to localization of light was realized using disordered photonic lattices [3-7], in which light propagates freely along one axis, and exhibits localization in the transverse directions ("transverse" localization [8]). The equations describing the propagation of light in these systems are identical to the equations describing the evolution of a single quantum particle in an atomic lattice, under the tight-binding approximation, thus allowing for the direct observation of Anderson localization as originally described in [1]. In these experiments, a localized wave packet, typically a single site wide, was released inside the disordered lattice and allowed to expand. In periodic lattices, such experiments led to the observation of ballistic wave-packet expansion $[9,10]$. As a result of the disorder the wave packet exhibited a modified expansion profile, the features of which depend on the dimensionality of the system [4,5], eventually settling to an exponentially localized distribution: the hallmark of Anderson localization. Recently, a similar approach enabled the direct observation of Anderson localization of matter waves [11-13] in disordered optical potentials, also described by the same equations [14].

While it is well established that in Anderson localization the average density distribution exhibits exponential localization, not much is known about higher correlations of the localized fields. Here we show that spatial intensity correlations, also known as Hanbury Brown and Twiss correlations [15], do not necessarily display a uniform decay. Moreover, in contrast to the density distribution, we find that these correlations carry a signature of the type of disorder: While light localized in lattices with on-site (diagonal) disorder show decaying correlations, the correlations in lattices with random tunneling amplitudes (off-diagonal disorder) exhibit oscillations. We relate the observed correlation features to a spectral symmetry that exists in lattices with off-diagonal disorder and is absent in lattices with diagonal disorder. Specifically, the eigenvalues in these disordered lattices are antisymmetrically distributed about the mean value, and eigenmodes associated with symmetric eigenvalues share several properties. These results are related to a recent prediction of quantum correlations in the single particle limit [16], and we now show that some analogous features can be also be observed in the classical regime.

The description for Anderson localization of light in onedimensional waveguide lattices is given by set of coupled discrete Schrodinger equations

$$
-i \frac{\partial U_{n}}{\partial z}=\beta_{n} U_{n}+C_{n, n+1} U_{n+1}+C_{n, n-1} U_{n-1} .
$$

Here $n=1, \ldots, N$ where $N$ is the number of lattice sites (waveguides), $U_{n}$ is the wave amplitude at site $n, \beta_{n}$ is the eigenvalue (propagation constant) associated with the $n$th site, $C_{n, n \pm 1}$ are the tunneling amplitudes between two adjacent sites, and $z$ is the longitudinal space coordinate (for a more detailed description see, e.g., [5]). These equations are identical to the equations describing the time evolution of a single electron in a lattice under the tight-binding approximation $[9,10]$, where $z$ represents time and $U_{n}$ is the wave function at site $n$. Therefore, while the experiments described in this Rapid Communication were conducted in the optical domain, the results hold also for other systems described by the tight-binding model, such as an electron in crystalline structure or Bose-Einstein condensate in disordered optical potentials.

In the tight-binding model, the disorder type falls into two broad categories: diagonal disorder, in which the $\beta_{n}$ parameters are randomized, but the tunneling amplitudes $C_{n, n \pm 1}$ are fixed across the lattice. Such disorder was considered by Anderson 

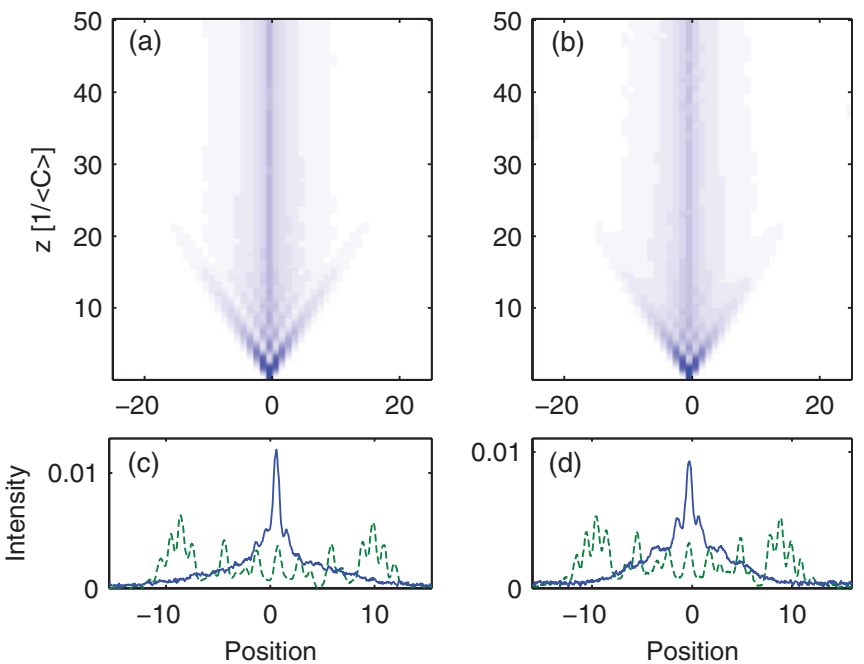

FIG. 1. (Color online) Observation of Anderson localization in lattices with diagonal and off-diagonal disorders. Top panels show simulation of the average wave-packet expansion in lattices with (a) diagonal and (b) off-diagonal disorder, when excited at a single site, showing similar dynamics. (c, d) Experimental measurements of the output distributions for the two types of disorder (blue, solid lines) as compared to the same distribution in a periodic lattice (green, dashed lines).

in his original work [1], in what is now known as the Anderson model. With few exceptions [6,7], all the recent experiments reporting the observation of Anderson localization of light [4,5] and matter waves [11,12] were conducted using this type of disorder. A second type of theoretically well-studied disorder is known as "off-diagonal" disorder, in which the $\beta_{n}$ parameters are fixed across the lattice, yet the tunneling amplitudes are randomized. Such lattices are known to exhibit several unique spectral properties [17-21]. However, very little has been achieved so far to experimentally observe a signature of these properties.

The recent experiments on Anderson localization of light [3-5] and matter waves [11,12], have reported the direct measurement of the main features of localization, namely the crossover from ballistic transport to localization as a function of time and the level of disorder. For example, Fig. 1 shows simulations of the wave-packet dynamics in disordered onedimensional lattices [5]. This dynamics starts with a ballistic expansion of the wave packet, similarly to the expansion in perfectly periodic lattices. After some propagation, a localized component emerges nearthe origin, coexisting with the transient, ballistic component. As the waves propagate, the ballistic component decays and the intensity distribution becomes exponentially localized in space. Figure 1 compares this evolution in lattices with diagonal [Fig. 1(a)] and off-diagonal [Fig. 1(b)] disorders, showing practically identical evolution.

We have fabricated two types of disordered lattices of waveguides, similar to those used to demonstrate Anderson localization of light [5]. In one array, the waveguide widths were identical, but their separations were randomized, modeling off-diagonal disorder. The other, a diagonally disordered array, had random-width waveguides yet constant separations. Light was launched into individual waveguides and the output (a) Diagonal disorder

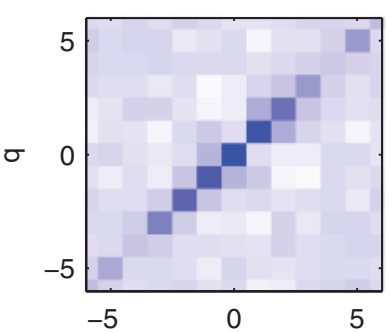

(c) Off-diagonal disorder

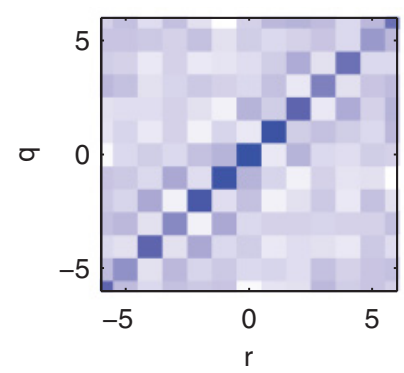

(b)

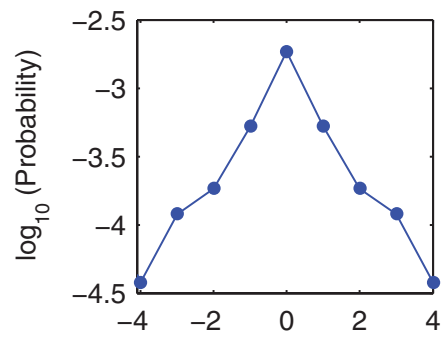

(d)

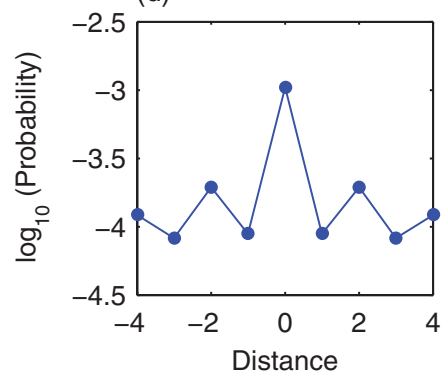

FIG. 2. (Color online) Measured density-density correlations $\Gamma_{r, q}=\left\langle I_{r} I_{q}\right\rangle /\left\langle I_{r}\right\rangle\left\langle I_{q}\right\rangle$ for localized wave packets in (a) lattices with diagonal disorder. The strong diagonal feature reflects finite coherence length of the waves (see text). (b) The extracted correlation function. (c) Density correlations in lattices with off-diagonal disorder, showing checker-like correlations. (d) The extracted correlation function, showing oscillating correlations.

distribution was recorded. When the output intensity was averaged over $\sim 100$ realizations (by launching the light at different input locations) we reproduced the exponentially decaying localization shown in Figs. 1(c) and (d). It is shown in comparison with the ballistic expansion that was measured in perfectly periodic arrays. We note that the two types of disorder led to very similar localized states.

A significant difference was observed, however, when we measured the density correlations of the output distribution. Figure 2 presents experimental results of density-correlation measurements in disordered lattices. Here, for each realization of disorder, the density autocorrelation is measured, and then averaged over many realizations (again by shifting the input site). The result is then normalized, so that $\Gamma_{r, q}=$ $\left\langle I_{r} I_{q}\right\rangle /\left\langle I_{r}\right\rangle\left\langle I_{q}\right\rangle$. As can be seen in Fig. 2, both types of disorder show a distinct diagonal feature in the correlation matrix. The length scale of this feature along the main diagonal $(q=r)$ of the matrix is the localization length. The width of the diagonal feature is given by the correlation length, which is not represented in the ensemble-averaged density distributions. In each single realization, the density distribution is not a smooth exponentially decaying distribution; it is speckled [4,5]. The width of the diagonal feature reflects the average speckle size. Since in each realization the speckles patter varies, their features are smeared out in the averaged distribution, and the information about their width is lost. However, the fact that these speckles have a characteristic length scale is recorded in the averaged correlation function.

We find that the correlations function carried additional information on the type of disorder in the lattice, information that is also lost when one considers the average density 
distributions. A closer look at the correlation matrix reveals that for lattices with off-diagonal disorder [Fig. 2(c)] the correlations tend to form a checkered pattern. This can be better seen when looking at the correlation function $g(\Delta r)$, extracted from the correlation matrix $\Gamma_{r, q}$ by summing over the diagonals, $g(\Delta r)=\sum_{r} \Gamma_{r, r+\Delta r}$. The density correlation decays smoothly for lattices with diagonal disorder, yet it exhibits decaying oscillations for lattices with off-diagonal disorder. These results were corroborated in numerical simulations (not shown).

To explain the dependence of the density correlations on the type of disorder, we start by pointing out a symmetry that exists in periodic lattices that is sustained also in lattices with pure off-diagonal disorder, but not in lattices with diagonal disorder [20-23]. Without loss of generality, we can set the diagonal terms of the Hamiltonian to zero, and we denote the randomized tunneling terms as $C_{n}$. Let $\left(a_{1}, \ldots, a_{N}\right)$ be an eigenvector with an eigenvalue $\lambda$. Then $C_{1} a_{2}=\lambda a_{1}$; $C_{1} a_{1}+C_{2} a_{3}=\lambda a_{2} ; \quad C_{2} a_{2}+C_{3} a_{4}=\lambda a_{3}$, and so on. Now it is obvious that the vector $b_{n}=(-1)^{n} a_{n}$, is also an eigenvector with an eigenvalue $-\lambda$. That is, the eigenvectors are paired around the center of the band, where twin eigenmodes share the same density distribution in absolute value, but an opposite (staggered) phase structure. It is also easy to see that this property will not be exhibited by lattices with diagonal disorder. For a more detailed proof, see [24].

To visualize this symmetry, we show in Fig. 3 the eigenvalues and eigenmodes for a single realization of a disordered lattice with diagonal versus a lattice with off-diagonal disorder. In Figs. 3(a) and 3(b), we compare the spectrum ("band") of eigenvalues for lattices with $N=50$ sites. As was shown for lattices with diagonal disorder [5], disordered lattices support two types of tightly localized eigenmodes with eigenvalues at the edges of the spectral band. At one edge the eigenmodes are tightly localized in space, each mode occupies a different location, and they generally have a flat phase profile (zero phase difference between adjacent sites). At the other edge of the spectrum the modes are also tightly localized, only they are staggered: there is a $\pi$ phase difference between adjacent sites. The eigenmodes at the center of the band have a more complicated phase structure, and they are typically wider. As we concluded above, in lattices with off-diagonal disorder we find that each eigenmode at one edge of the spectrum had a twin eigenmode at the other edge. These twin eigenmodes occupy the same region of the lattice and have the same distribution of the absolute amplitude, but they differ in phase structure as shown in Fig. 1(c). This property does not exist in lattices with diagonal disorder [see Fig. 1(d)].

This special symmetry of the spectrum in the off-diagonal model can be observed experimentally. Briefly, it is possible to excite pure localized eigenmodes by using either a flat-phase or a staggered beam, with the correct width and initial position at the lattice. Flat-phased and staggered localized eigenmodes of a lattice with diagonal disorder were measured (see Fig. 2 in [5]), showing indeed that in diagonally disordered lattices the positions of the lowest-eigenvalues flat-phased eigenmodes never coincided with the position of the highest-eigenvalues, staggered localized modes. Figure 3(f) shows an excitation of a flat-phased eigenmode in a lattice with diagonal disorder. Indeed, when the same input beam was tilted to excite neighbor-
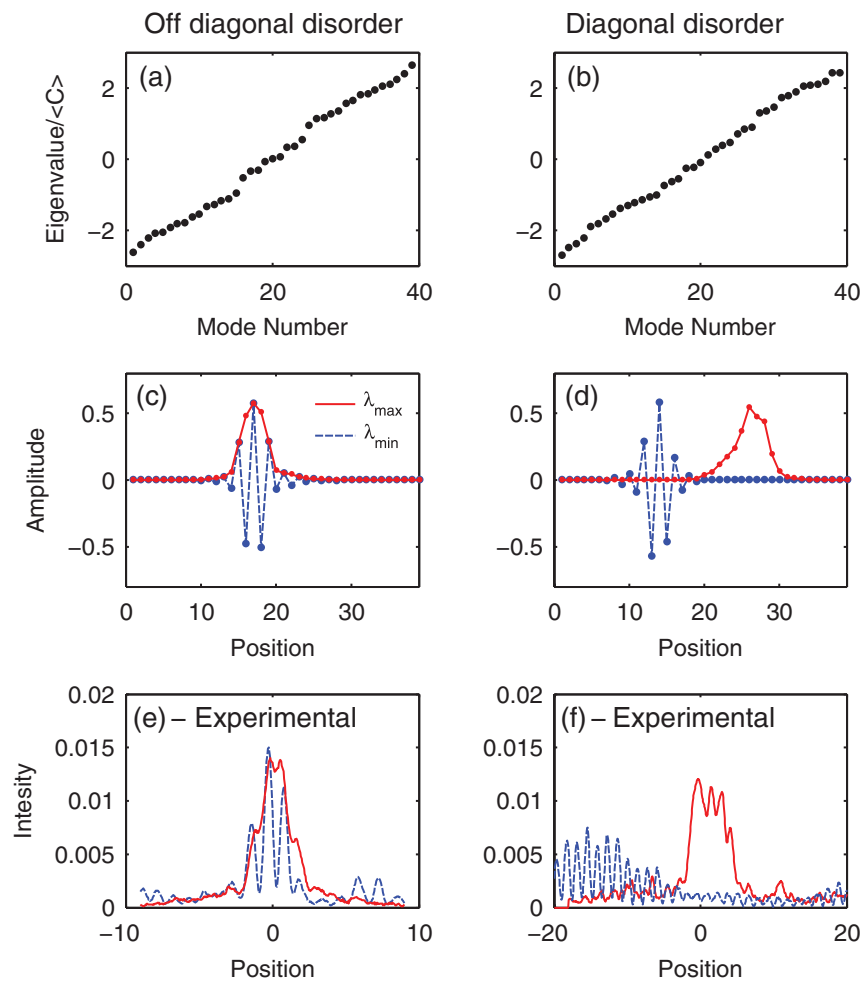

FIG. 3. (Color online) Spectral symmetry in disordered lattices. (a) The spectrum (band) of eigenvalues for lattices with off-diagonal disorder. Note the symmetry in the eigenvalues with respect to zero, for every eigenvalue $\lambda$ there exists a counterpart eigenvalue $\tilde{\lambda}=(-\lambda)$ (see text). (b) In lattices with diagonal disorder no exact symmetry exists in the spectrum of eigenvalues. (c) An example of the spatial distribution of a pair of eigenmodes in the off-diagonal case that have symmetric eigenvalues [in this example the extreme eigenvalues in the system $\lambda_{\max }$ (solid line) and $\lambda_{\min }$ (dashed)]. The two eigenmodes occupy the same region of the lattice and have the same spatial distribution, but vary in phase structure. A similar result can be observed for all other symmetric pairs. (d) In this diagonal disorder case, the eigenmodes associated with opposite eigenvalues occupy different regions of the lattice. (e) Experimental measurement of two spectrally symmetric eigenmodes in a lattice with off-diagonal disorder, showing their spatial similarity. (f) Measurement showing that in the diagonal disorder case the same location in the lattice cannot host two spectrally symmetric localized modes (see text).

ing sites with a $\pi$ phase difference, the output density showed considerable expansion, suggesting that no staggered localized eigenmode resides in the same location. In contrast, Fig. 3(e), shows the same procedure in a lattice with off-diagonal disorder. Here, the flat-phased beam excited a flat-phased, localized eigenmode, and a beam with a $\pi$ phase difference between adjacent sites excited a staggered localized eigenmodes with the same width, the same spatial profile, and at the same location.

Now, to explain the different density-density correlations shown in Fig. 2, we need to consider the effect of these different spectral properties on the expanding wave packets when they are excited at a single site. The localization effect arises from the fact that all the eigenmodes that are excited have a finite extent. It is well known that in infinite one-dimensional disordered systems all eigenmodes of the system are localized. In lattices with off-diagonal disorder, an initial excitation 
of a single lattice site necessarily involves the simultaneous excitations of pairs of "twin" eigenmodes, as they have identical overlap with the initially excited site. The sum of two identical amplitude distributions yet with a $\pi$-phase difference in each second site results in a density comb-like pattern that nulls at every second site. In the dynamic problem several pairs could be excited simultaneously by the single site initial condition, and the two modes of the pair accumulate phase in a different rate (according to their eigenvalues). Nevertheless, the wave packet will contain a component with an oscillating intensity pattern, with a spatial frequency of two sites. This effect is washed out in the density distribution averaged over all realizations of disorder, as in each realization the oscillations appear in a different location. However, the fact that such oscillations appear in each realization will be recorded in the averaged correlation.

We note a previous publication by our group [16], in which oscillating quantum correlations were analyzed theoretically for bosonic or fermionic pairs, predicting checker-like correlations in some cases. Those phenomena are not unrelated to the results reported here, yet the oscillating correlations reported there were of quantum origin (i.e., in the case of light they require the use of nonclassical light), while here the described effects are purely classical wave effects.

In conclusion, we have experimentally shown that density correlation measurements can carry a signature of the type of disorder that exists in a given sample, and we have traced that signature to the existence of a unique spectral symmetry that is exhibited by lattices with off-diagonal disorder. Similar results can be measured in matter waves systems, using density-correlation measurements [25] in disordered lattices. It might also be interesting to study the effect of nonlinear interactions on these correlations, either in the optical or matter wave experiments. The signature of these results might also be observed in correlations measurements for multiply scattered classical [26] and nonclassical [27,28] light, and in lattices with other types of symmetries [29].

This work was supported by the German-Israel Foundation (GIF), the Minerva Foundation, and the Crown Photonics Center. YL acknowledges support from the Israeli Academy of Science and Humanities.
[1] P. W. Anderson, Phys. Rev. 109, 1492 (1958).

[2] P. A. Lee and T. V. Ramakrishnan, Rev. Mod. Phys. 57, 287 (1985).

[3] T. Pertsch et al., Phys. Rev. Lett. 93, 053901 (2004).

[4] T. Schwartz et al., Nature (London) 446, 52 (2007).

[5] Y. Lahini, et al. Phys. Rev. Lett. 100, 013906 (2008).

[6] H. B. Perets et al., Phys. Rev. Lett. 100, 170506 (2008).

[7] A. Szameit et al., Opt. Lett. 35, 1172 (2010).

[8] H. De Raedt, A. Lagendijk, and P. de Vries, Phys. Rev. Lett. 62, 47 (1989).

[9] D. N. Christodoulides, F. Lederer, and Y. Silberberg, Nature (London) 424, 817 (2003).

[10] F. Lederer et al., Phys. Rep. 463, 1 (2008).

[11] J. Billy et al., Nature (London) 453, 891 (2008).

[12] G. Roati et al., Nature (London) 453, 895 (2008).

[13] J. Chabe et al., Phys. Rev. Lett. 101, 255702 (2008).

[14] G. Modugno Rep. Prog. Phys. 73, 102401 (2010).

[15] R. Hanbury Brown and R. Q. Twiss, Nature (London) 177, 27 (1956).

[16] Y. Lahini, Y. Bromberg, D. N. Christodoulides, and Y. Silberberg, Phys. Rev. Lett. 105, 163905 (2010).

[17] F. J. Dyson, Phys. Rev. 92, 1331 (1953).
[18] C. M. Soukoulis and E. N. Economou, Phys. Rev. B 24, 5698 (1981).

[19] C. M. Soukoulis, I. Webman, G. S. Grest, and E. N. Economou, Phys. Rev. B 26, 1838 (1982).

[20] A. Komiyama, IEICE Trans. Electron. E83, 736 (2000).

[21] M. Inui, S. A. Trugman, and E. Abrahams, Phys. Rev. B 49, 3190 (1994).

[22] F. Wegner, Z. Phys. B 44, 9 (1981).

[23] C. Mudry, P. W. Brouwer, and A. Furusaki, Phys. Rev. B 62, 8249 (2000).

[24] See Supplemental Material at http://link.aps.org/supplemental/ 10.1103/PhysRevA.84.041806 for the full proof.

[25] E. Altman, E. Demler, and M. D. Lukin, Phys. Rev. A 70, 013603 (2004).

[26] E. Akkermans and G. Montambaux, Mesoscopic Physics of Electrons and Photons (Cambridge University Press, Cambridge, England, 2007).

[27] S. Smolka, A. Huck, U. L. Andersen, A. Lagendijk, and P. Lodahl, Phys. Rev. Lett. 102, 193901 (2009).

[28] J. R. Ott, N. A. Mortensen, and P. Lodahl, Phys. Rev. Lett. 105, 090501 (2010).

[29] Y. N. Joglekar, Phys. Rev. A 82, 044101 (2010). 